\title{
Survival analysis and postoperative complications after ventricular assist device implantation; prognostic value of INTERMACS scale.
}

\author{
Ahmed R. Ahmed ${ }^{1}$, Pizanis Nikolaus ${ }^{1}$, Koch Achim¹, Carstens Henning ${ }^{1}$, Rassaf Tienush², Ludike \\ Peter $^{2}$, Kamler Markus ${ }^{1^{*}}$ \\ ${ }^{1}$ Department of Thoracic and Cardiovascular Surgery, University Hospital Essen, Essen, Germany \\ ${ }^{2}$ Department of Cardiology and Vascular Medicine, University Hospital Essen, Essen, Germany
}

\begin{abstract}
Background: Ventricular assist devices (VADs) improve survival and quality of life in patients with refractory heart failure. This study retrospectively evaluates patients' profiles, clinical outcome in patients who underwent LVAD implantation in regard to their INTERMACS level.

Methods: In a single center analysis, data of 104 patients after LVAD implantation between August 2010 and March 2015 were analyzed retrospectively. According to INTERMACS level, 3 groups were compared: Group A (INTERMCAS level 1, n=27 patients), group B (INTERMACS level 2/3, $n=47$ ) and group $C$ (INTERMACS level 4-6, n=30). Preoperative parameters and postoperative outcome were compared between groups.

Results: Mortality was higher in group A compared to group $B(p=0.017)$ and group $C(p=0.017)$. Sepsis was the most common post-operative complication after LVAD implantation.

Conclusion: INTERMACS level was related to outcome after LVAD implantation. Optimization of preoperative volume status, preload and sepsis management are recommended to lower mortality in these patients.
\end{abstract}

Keywords: Left Ventricular Assist Device (LVAD), Heart Ware Ventricular Assist device (HVAD), INTERMACS.

\section{Introduction}

Patients with end stage heart failure under optimized medical therapy have a very poor prognosis (REMATCH) [1]. Cardiac transplantation provides considerable survival benefits for patients with end-stage heart failure; however, its use is limited due to worldwide shortage of donor organs $[2,3]$. Therefore, implantable left ventricular assist device (LVAD) therapy is a promising alternative for patients awaiting cardiac transplantation (bridge to transplantation (BTT)], for patients who are ineligible for transplantation (destination therapy (DT)) or who are expected to recover after left ventricular unloading (bridge to recovery (BTR)).
Impact of patient selection on outcome of LVAD surgery has been recognized since implantation of the first devices. Regardless of the type of device, use of VADs in patients with severe functional impairment, end-organ dysfunction and right ventricular failure, malnutrition, or infection had been consistently associated with unfavorable outcome [4-6]. To allow for risk stratification, the Interagency Registry for Mechanically Assisted Circulatory Support (INTERMACS) investigators developed a classification based on severity of illness at the time of VAD implant ranging from critical cardiogenic shock to stable ambulatory heart failure (Table 1). The scale facilitates communication between colleagues and refines patient selection for optimizing outcomes [7,8].

\begin{tabular}{|c|c|c|}
\hline Profiles & Brief Description & Details \\
\hline INTERMCAS 1 & Critical cardiogenic shock (Crash and burn) & $\begin{array}{l}\text { Life threatening hypotension and critical organ hypoperfusion despite rapidly } \\
\text { escalating inotropic support. }\end{array}$ \\
\hline INTERMACS 2 & Progressive decline (sliding fast on inotrope) & $\begin{array}{l}\text { Declining function despite inotropic support, manifested by worsening renal function, } \\
\text { nutritional depletion, inability to restore volume balance }\end{array}$ \\
\hline INTERMACS 3 & $\begin{array}{l}\text { Stable but inotrope dependent (Dependent } \\
\text { stability) }\end{array}$ & $\begin{array}{l}\text { Patient with stable blood pressure, organ function on continuous intravenous inotropic } \\
\text { support but demonstrating repeated failure to wean from support }\end{array}$ \\
\hline INTERMACS 4 & Resting symptoms on oral therapy at home & $\begin{array}{l}\text { Patient can be stabilized close to normal volume status but experiences daily } \\
\text { symptoms of congestion at rest or during ADL. }\end{array}$ \\
\hline INTERMACS 5 & Exertion intolerant & $\begin{array}{l}\text { Comfortable at rest and with ADL without congestive symptoms but unable to engage } \\
\text { in any other activity, living predominantly within the house. }\end{array}$ \\
\hline INTERMCAS 6 & Exertion limited (Walking wounded) & $\begin{array}{l}\text { Patient without evidence of fluid overload is comfortable at rest, and with activities of } \\
\text { daily living and minor activities outside the home but fatigues after the first few minutes } \\
\text { of any meaningful activity. }\end{array}$ \\
\hline INTERMACS 7 & Advanced NYHA class III (Placeholder) & A placeholder for more precise specification in future. \\
\hline
\end{tabular}


It was the aim of this study to retrospectively evaluate preoperative patients' profiles, clinical outcome, postoperative complications and survival in patients who underwent LVAD implantation considering the INTERMACS level.

\section{Methods}

\section{Patients and data collection}

Data of 104 adult patients with end stage heart failure admitted to our institution between August 2010 and March 2015 were analyzed. Data were collected from our center database. We retrospectively analyzed preoperative demographic data, clinical, hemodynamic and laboratory parameters as well as the implant strategy. Furthermore, we evaluated postoperative complications and mortality. Patients were selected for LVAD implantation after an interdisciplinary evaluation of disease status and upon completing the preoperative diagnostic procedures. Patients were categorized per INTERMACS classification at the time of implant. Preoperative INTERMACS profiles were as follows: level 1 in 27 patients, level 2 in 20 patients, level 3 in 27 patients, level 4 in 25 patients, level 5 in 4 patients and level 6 in 1 patient respectively. Patients were then classified into 3 groups based on similarity of clinical symptoms; group A (cardiogenic shock) included 27 patients at INTERMACS level 1, group B (inotrope-dependent and hospitalized) included 47 patients at INTERMACS level 2/3 and group $\mathrm{C}$ (outpatients with low functional capacity) included 30 patients INTERMACS level 4/5/6.

\section{Device implantation}

All device implants were 3rd generation continuous flow, centrifugal blood pumps type Heart Ware ventricular Assist device HVAD ${ }^{\circledR}$ (Heart Ware, Inc., Framingham, Massachusetts). The implantation strategies were destination therapy (DT) in $79.8 \%$ ( $\mathrm{n}=83$ patients), followed by bridge to transplantation (BTT) $(16.3 \% \mathrm{n}=17$ patients) and bridge to recovery (BTR) in 4 patients $(3.8 \%)$.

\section{Postoperative outcome}

Analysis included duration of VAD support, total length of stay
(LOS) in the hospital and intensive care unit, days on inotropes, days on mechanical ventilation. Patient records were examined for common LVAD-associated complications. Definitions are classified in (Table 2). Date of onset was recorded for all adverse events to conduct time-to-event analysis. Total, early (at 30 days after implantation) and late (after 30 days after implantation) mortality as well as its causes and preoperative predictors of total mortality were analyzed. Survival analysis included survival in days on mechanical circulatory support, survival to hospital discharge or to end of the study (31.03.2015) and survival rates at 3 months, 6 months and 1 year.

\section{Ethics}

This study was approved by the local ethics committee.

\section{Statistical analysis}

All data were presented as mean $\pm \mathrm{SD}$ unless indicated elsewhere. Continuous data were evaluated for normality using one-sample Kolmogorov-Smirnov test. Univariate analysis was performed using either Student's t-test or Mann-Whitney U test for normal and non-normal continuous variables, respectively. Pearson's $\chi 2$ or Fisher exact tests were used for categorical data dependent on the minimum expected count in each cross tab. Kaplan-Meier actuarial survival estimate was generated to analyze post-LVAD survival of the entire cohort. All data were analyzed using Statistical Package for Social Sciences, version 20.0 (SPSS Inc., Chicago, IL) and are expressed as the mean \pm standard deviation in case of normal distributed or median (interquartile range) in case of non-normal distributed continuous variables. The categorical data are expressed as total numbers and percentages.

\section{Results}

Baseline characteristics of the enrolled patients are shown in (Table 3). Most of the patients enrolled were implanted as destination therapy (79.8\% of patients). Pre-operative hemodynamics and clinical profiles are summarized in (Table 4). Although left ventricular ejection fraction before LVAD implant was $<30 \%$ in all groups, hemodynamic differences were considerable. Group A had a significantly lower mean arterial pressure than group B

Table 2. Definitions of postoperative complications ${ }^{[10-14]}$.

\begin{tabular}{|c|c|}
\hline Postoperative complication & Definition \\
\hline Sepsis & Presence of SIRS plus a documented source of infection \\
\hline Acute respiratory failure & $\begin{array}{l}\mathrm{pO} 2<60 \mathrm{mmHg} \text { and or } \mathrm{pCO} 2>50 \mathrm{mmHg} \text { or difficult weaning from mechanical ventilation at any time or inability to discontinue } \\
\text { ventilator support after six or more days of VAD support. }\end{array}$ \\
\hline Acute liver injury & $\begin{array}{l}\text { 3-fold increase in the transaminases versus the value before VAD or total bilirubin levels elevated three times baseline values } \\
\text { for } \geq \text { seven days. }\end{array}$ \\
\hline Acute renal failure & $\begin{array}{l}\text { at least } 2 \text {-fold increase in the serum creatinine or glomerular filtration rate decrease }>50 \% \text {, compared with the pre-operative } \\
\text { level, or urine output }<0.5 \mathrm{ml} / \mathrm{kg} / \mathrm{h} \text { for } 6 \text { hours or new renal replacement therapy. }\end{array}$ \\
\hline Right heart failure & $\begin{array}{l}\text { clinical parameters of low LVAD output, and high central venous pressure ( }>15 \mathrm{~mm} \mathrm{Hg} \text { ), and the use of inhaled nitric oxide }>48 \\
\text { hours, inotropic support }>14 \text { days, or need for RVAD after LVAD implantation }\end{array}$ \\
\hline Hemorrhage & $\begin{array}{l}\text { Bleeding resulted in death or the need for re-operation, hospitalization, or transfusions of red blood cells ( } \geq 4 \text { units PRBC within } \\
\text { any } 24 \text { hours' period in the first } 7 \text { days' post-implant or } \geq 2 \text { units PRBC within any } 24 \text { hours' period after } 7 \text { days' post-implant). }\end{array}$ \\
\hline Neurological event & A new onset ischemic or hemorrhagic stroke documented by CT-Brain. \\
\hline Hemolysis & plasma free hemoglobin $>40 \mathrm{mg} / \mathrm{dl}$ in association with clinical signs of hemolysis \\
\hline Multiorgan failure & presence of a systemic inflammatory response (SIRS criteria) and dysfunction of at least 2 organs \\
\hline Psychological event & Severe disturbance in thinking, emotion, or behavior requiring intervention. \\
\hline Wound healing disturbance & disruption of the apposed surfaces of the surgical incision, requiring surgical repair and or VAC therapy \\
\hline Device failure & $\begin{array}{l}\text { Divided into } 3 \text { categories: } 1 \text {. Pump thrombosis defined as an obstructive thrombus in the device associated with clinical } \\
\text { symptoms of impaired pump performance or the need for thrombolytic or surgical intervention. } 2 \text {. Driveline disconnection } 3 . \\
\text { Device malfunction }\end{array}$ \\
\hline
\end{tabular}


Table 3. Baseline characteristics of the study cohort before LVAD implantation, split by INTERMACS level (group A: level 1, group B: level 2/3, group C: level 4/5/6).

\begin{tabular}{|c|c|c|c|c|c|c|c|}
\hline Baseline characteristic & Cohort $(n=104)$ & GroupA $(n=27)$ & Group B $(n=47)$ & Group C $(n=30)$ & $p$ value $A$ vs $B$ & $p$ value $A$ vs $C$ & $p$ value $B$ vs $C$ \\
\hline Age (in years) & 59.1 & 55.7 & 58.91 & 62.33 & \multirow{2}{*}{0.271} & \multirow{2}{*}{0.055} & \multirow{2}{*}{0.217} \\
\hline Mean \pm SD & \pm 11.3 & \pm 12.8 & \pm 11.2 & \pm 9.22 & & & \\
\hline Male (\%) & $76.9 \%$ & $70.4 \%$ & $74.5 \%$ & $86.7 \%$ & 0.788 & 0.195 & 0.198 \\
\hline $\mathrm{BSA}\left(\mathrm{m}^{2}\right)$ & 1.96 & 1.97 & 1.93 & 1.99 & \multirow{2}{*}{0,669} & \multirow{2}{*}{0.497} & \multirow{2}{*}{0.242} \\
\hline Mean \pm SD & $\pm 0,22$ & \pm 0.24 & \pm 0.21 & \pm 0.22 & & & \\
\hline $\mathrm{BMI}(\mathrm{kg} / \mathrm{m} 2)$ & 28.76 & 25.47 & 25.34 & 28.07 & \multirow{2}{*}{0.999} & \multirow{2}{*}{0.02} & \multirow{2}{*}{0.004} \\
\hline Mean \pm SD & \pm 3.71 & \pm 3.37 & \pm 3.44 & \pm 3.82 & & & \\
\hline
\end{tabular}

Table 4. Preoperative clinical and hemodynamic parameters, also split by INTERMACS level (group A: level 1, group B: level 2/3, group C: level $4 / 5 / 6)$.

\begin{tabular}{|c|c|c|c|c|c|c|c|}
\hline Parameter & Cohort $(n=104)$ & Group A (n=27) & Group B $(n=47)$ & Group C (n=30) & $p$ value $A$ vs $B$ & $p$ value $A$ vs $C$ & $p$ value $B$ vs $C$ \\
\hline $\mathrm{CVP}(\mathrm{mmHg})$ & 14.39 & 14.55 & 14.76 & 13.66 & \multirow{2}{*}{0.999} & \multirow{2}{*}{0.999} & \multirow{2}{*}{0.992} \\
\hline Mean \pm SD & \pm 4.79 & \pm 4.33 & \pm 5.45 & \pm 4.08 & & & \\
\hline UOP (ml/h) & 94.8 & 80.1 & 98.63 & 102.24 & \multirow{2}{*}{0.617} & \multirow{2}{*}{0.507} & \multirow{2}{*}{0.999} \\
\hline Mean \pm SD & \pm 60.30 & \pm 71.78 & \pm 56.66 & \pm 54.06 & & & \\
\hline MAP (mmHg) & 70.69 & 64.05 & 72.91 & 73.2 & \multirow{2}{*}{0.001} & \multirow{2}{*}{0.001} & \multirow{2}{*}{0.999} \\
\hline Mean \pm SD & \pm 8.84 & \pm 10.33 & \pm 6.77 & \pm 7.32 & & & \\
\hline Mechanical ventilation (\%) & 25 & 77.8 & 10.6 & 0 & 0.001 & 0.001 & 0.085 \\
\hline Inotropic support (\%) & 71.2 & 100 & 100 & 0 & 0.999 & 0.001 & 0.001 \\
\hline CVVHD (\%) & 29.8 & 55.6 & 27.7 & 0 & 0.025 & 0.001 & 0.001 \\
\hline ECMO (\%) & 16.3 & 63 & 0 & 0 & 0.001 & 0.001 & 0.001 \\
\hline IABP (\%) & 20.2 & 48.1 & 17 & 0 & 0.007 & 0.001 & 0.02 \\
\hline $\operatorname{COP}(\mathrm{L} / \mathrm{min})$ & 3.59 & 3.23 & 3.66 & 3.81 & \multirow{2}{*}{0.279} & \multirow{2}{*}{0.121} & \multirow{2}{*}{0.999} \\
\hline Mean \pm SD & \pm 1.06 & \pm 0.92 & \pm 1.07 & \pm 1.09 & & & \\
\hline $\mathrm{Cl}(\mathrm{L} / \mathrm{min} / \mathrm{m} 2)$ & 1.9 & 1.67 & 1.92 & 2.07 & \multirow{2}{*}{0.173} & \multirow{2}{*}{0.02} & \multirow{2}{*}{0.743} \\
\hline Mean \pm SD & \pm 0.55 & \pm 0.38 & \pm 0.56 & \pm 0.61 & & & \\
\hline SVR & 1361.59 & 1455.61 & 1380.19 & 1247.86 & \multirow{2}{*}{0.512} & \multirow{2}{*}{0.163} & \multirow{2}{*}{0.255} \\
\hline$\left(\right.$ Dyn.sec/cm $\left.{ }^{5}\right)$ Mean \pm SD & \pm 449.70 & \pm 475.82 & \pm 474.54 & \pm 368.68 & & & \\
\hline LVEF (\%) & 17.08 & 16.59 & 16.09 & 19.05 & \multirow{2}{*}{0.258} & \multirow{2}{*}{0.252} & \multirow{2}{*}{0.033} \\
\hline Mean \pm SD & \pm 5.82 & \pm 4.59 & \pm 6.09 & \pm 6.06 & & & \\
\hline Systolic PAP & 48.65 & 47.14 & 49.04 & 49.4 & \multirow{2}{*}{0.999} & \multirow{2}{*}{0.999} & \multirow{2}{*}{0.999} \\
\hline$(\mathrm{mmHg})$ Mean $\pm \mathrm{SD}$ & \pm 16.34 & \pm 15.50 & \pm 17.42 & \pm 15.76 & & & \\
\hline Mean PAP & 30.18 & 26.66 & 31.44 & 31.36 & \multirow{2}{*}{0.233} & \multirow{2}{*}{0.342} & \multirow{2}{*}{0.999} \\
\hline $\mathrm{mmHg}$ Mean $\pm \mathrm{SD}$ & \pm 11.19 & \pm 10.98 & \pm 11.97 & \pm 9.70 & & & \\
\hline PCWP & 22.77 & 20.59 & 23.89 & 23 & \multirow{2}{*}{0.402} & \multirow{2}{*}{0.956} & \multirow{2}{*}{0.999} \\
\hline$(\mathrm{mmHg})$ Mean $\pm \mathrm{SD}$ & \pm 9.06 & \pm 6.91 & \pm 10.72 & \pm 7.75 & & & \\
\hline PVR & 219.09 & 210.63 & 229.45 & 210.48 & 0.567 & 0818 & 0398 \\
\hline (Dyn.sec/cm5) Mean \pm SD & \pm 139.68 & \pm 122.89 & \pm 134.85 & \pm 163.09 & 0.561 & 0.818 & 0.398 \\
\hline RVSWI & 18.37 & 15.15 & 15.51 & 18.41 & 0.792 & 0.046 & 0.089 \\
\hline$\left(\mathrm{g} / \mathrm{m}^{2} /\right.$ beat $)$ Mean $\pm \mathrm{SD}$ & \pm 23.25 & \pm 4.90 & \pm 6.85 & \pm 6.77 & 0.192 & 0.040 & 0.009 \\
\hline
\end{tabular}

CVP: Central Venous Pressure; UOP: Urine Output; MAP: Mean Arterial Blood Pressure; CVVHD: Continuous Veno-Venous Hemodialysis; ECMO: Extracorporeal Membrane Oxygenation; IABP: Intra-Aortic Balloon Pump; COP: Cardiac Output; CI: Cardiac Index; SVR: Systemic Vascular Resistance; LVEF: Left Ventricular Ejection Fraction; PAP: Pulmonary Artery Pressure; PCWP: Pulmonary Capillary Wedge Pressure; PVR: Pulmonary Vascular Resistance; RVSWI: Right Ventricular Stroke Work Index.

and group $\mathrm{C}(\mathrm{p}<0.001)$. Cardiac index and right ventricular stroke work index were lower in group A than group $\mathrm{C}(\mathrm{p}=0.020$ and 0.046 respectively) whereas ejection fraction was lower in group $\mathrm{B}$ than $\mathrm{C}(\mathrm{p}=0.033) .77 .8 \%$ of patients in group $\mathrm{A}$ (patients $=21$ ) were mechanically ventilated in comparison with only 5 patients in group $B(10.6 \%)(p=0.001) .15$ patients $(55.6 \%)$ in group A required continuous veno-venous hemodialysis (CVVHD) in comparison with only $27.7 \%$ in group $B(p=0.025)$. Intra-aortic balloon pump (IBAP) therapy was significantly higher in group A $(48.1 \%)$ than group B $(17.0 \%)(p=0.007) .17$ patients in group A (63\%) received extracorporeal membrane oxygenation (ECMO) support. Pre-operative laboratory profiles of patients are listed in Table 5

\section{Postoperative complications}

The most common complication identified was sepsis (49 patients, $47.1 \%$ of the entire cohort), followed by right ventricular failure (39 patients, $37.5 \%$ of the cohort). Postoperative respiratory failure was also common and occurred in 35 patients $(33.7 \%), 23$ of them $(22.1 \%$ of the entire cohort) underwent tracheotomy. Renal failure occurred in $27(26.0 \%)$ patients; we excluded patients who underwent preoperative hemodialysis. Postoperative tachyarrhythmia occurred in 16 patients $(25 \%)$, with $1: 1$ ratio between supraventricular tachycardia (SVT) and ventricular tachycardia (VT). Twenty-one patients (20.2\%) suffered postoperative 
Citation: Ahmed AR, Pizanis N, Kamler M, et al. Survival analysis and postoperative complications after ventricular assist device implantation, prognostic value of INTERMACS scale. Ann Cardiovasc Thorac Surg. 2018;1(1):15-23.

Table 5. Preoperative laboratory parameters also split by INTERMACS level (group A: level 1, group B: level 2/3, group C: level 4/5/6).

\begin{tabular}{|c|c|c|c|c|c|c|c|}
\hline Laboratory profile & Cohort $(n=104)$ & Group A (n=27) & Group B $(n=47)$ & Group C (n=30) & $p$ value $A$ vs $B$ & $p$ value $A$ vs $C$ & pvalue $B$ vs $C$ \\
\hline Hemoglobin (g/dl) & 10.91 & 10.16 & 10.63 & 12.04 & \multirow{2}{*}{0.134} & \multirow{2}{*}{0.001} & \multirow{2}{*}{0.001} \\
\hline Mean \pm SD & \pm 2.18 & \pm 2.15 & \pm 2.05 & \pm 2.01 & & & \\
\hline Creatinine & 1.59 & 1.74 & 1.37 & 1.78 & \multirow{2}{*}{0.018} & \multirow{2}{*}{0.31} & \multirow{2}{*}{0.041} \\
\hline$(\mathrm{mg} / \mathrm{dl})$ Mean $\pm \mathrm{SD}$ & \pm 0.98 & \pm 0.73 & \pm 0.47 & \pm 1.56 & & & \\
\hline BUN & 29.16 & 38.8 & 24.72 & 27.43 & \multirow{2}{*}{0.01} & \multirow{2}{*}{0.086} & \multirow{2}{*}{0.238} \\
\hline$(\mathrm{mg} / \mathrm{dl})$ Mean $\pm \mathrm{SD}$ & \pm 17.98 & \pm 26.20 & \pm 12.52 & \pm 12.77 & & & \\
\hline GFR & 55.99 & 50.07 & 61.68 & 52.4 & \multirow{2}{*}{0.023} & \multirow{2}{*}{0.448} & \multirow{2}{*}{0.068} \\
\hline$(\mathrm{ml} / \mathrm{min})$ Mean $\pm \mathrm{SD}$ & \pm 20.54 & \pm 21.53 & \pm 19.19 & \pm 19.99 & & & \\
\hline Total bilirubin & 1.44 & 2.41 & 1.16 & 1.02 & \multirow{2}{*}{0.005} & \multirow{2}{*}{0.001} & \multirow{2}{*}{0.267} \\
\hline$(\mathrm{mg} / \mathrm{dl})$ Mean $\pm \mathrm{SD}$ & \pm 1.83 & \pm 2.87 & \pm 1.13 & \pm 1.12 & & & \\
\hline AST & 80.53 & 200.37 & 40.55 & 35.33 & \multirow{2}{*}{0.001} & \multirow{2}{*}{0.001} & \multirow{2}{*}{0.726} \\
\hline (U/litter) Mean \pm SD & \pm 167.44 & \pm 295.31 & \pm 41.99 & \pm 22.68 & & & \\
\hline ALT & 90.71 & 237.07 & 47.61 & 26.5 & \multirow{2}{*}{0.001} & \multirow{2}{*}{0.001} & \multirow{2}{*}{0.361} \\
\hline (U/Liter) Mean \pm SD & \pm 173.67 & \pm 281.13 & \pm 74.47 & \pm 15.79 & & & \\
\hline Platelet count $\left(\times 10^{9} /\right.$ liter $)$ & 214.44 & 141.25 & 248.55 & 226.86 & \multirow{2}{*}{0.001} & \multirow{2}{*}{0.001} & \multirow{2}{*}{0.655} \\
\hline Mean \pm SD & \pm 107.94 & \pm 88.90 & \pm 118.58 & \pm 70.44 & & & \\
\hline INR & 2.62 & 1.55 & 1.27 & 1.16 & \multirow{2}{*}{0.356} & \multirow{2}{*}{0.014} & \multirow{2}{*}{0.049} \\
\hline$($ Mean \pm SD) & \pm 13.41 & \pm 0.63 & \pm 0.49 & \pm 0.29 & & & \\
\hline PT & 70.6 & 59.03 & 69.31 & 83.01 & \multirow{2}{*}{0.38} & \multirow{2}{*}{0.022} & \multirow{2}{*}{0.021} \\
\hline (Seconds) Mean \pm SD & \pm 24.12 & \pm 21.95 & \pm 19.86 & \pm 26.88 & & & \\
\hline Lactate & 1.66 & 2.49 & 1.36 & 1.4 & \multirow{2}{*}{0.032} & 0009 & 0284 \\
\hline$(\mathrm{mmol} / \mathrm{L})$ Mean $\pm \mathrm{SD}$ & \pm 2.28 & \pm 4.19 & \pm 0.65 & \pm 1.11 & & 0.009 & 0.204 \\
\hline Metabolic acidosis (No.) & 12 & 9 & 2 & 1 & 0.001 & 0.003 & 0.999 \\
\hline$\%$ & $11.5 \%$ & $33.3 \%$ & $4.3 \%$ & $3.3 \%$ & 0.001 & 0.003 & 0.999 \\
\hline BNP & 1349.57 & 1462.48 & 1602.64 & 851.46 & 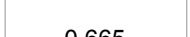 & 0170 & 0067 \\
\hline$(\mathrm{pg} / \mathrm{ml})$ Mean $\pm \mathrm{SD}$ & \pm 1458.17 & \pm 1347.38 & \pm 1800.13 & \pm 641.75 & 0.000 & 0.170 & $0.00 r$ \\
\hline PCT & 0.92 & 1.96 & 0.76 & 0.24 & 0.007 & 0.001 & 0.019 \\
\hline (ng/ml) Mean \pm SD & \pm 1.996 & \pm 2.70 & \pm 1.94 & \pm 0.25 & & & \\
\hline
\end{tabular}

BUN: Blood Urea Nitrogen. GFR: Glomerular Filtration Rate. BNP: Brain Natriuretic Peptide. ALT: Alanine Aminotransferase. AST: Aspartate Transferase. INR: International Normalizing Ratio. PT: Prothrombin Time. PCT: Procalcitonin.

surgery related hemorrhage; hemopericardium in 13 patients $(12.5 \%)$ patients and hemorrhagic pleural effusion in $8(7.7 \%)$ patients. Device related complications occurred in 11 patients representing $10.6 \%$ of the entire cohort, and were divided as follow; pump thrombosis (6 patients, 5.8\%), driveline disconnection (4 patients, $3.8 \%$ ) and device failure (1 patient, 1\%). (Figure. 1) Group A had a significant higher incidence of postoperative respiratory failure $(\mathrm{p}=0.013)$, surgery related hemorrhage $(p=0.029)$ and multiorgan failure $(p=0.014)$ than group $\mathrm{B}$ as well as a higher incidence of respiratory failure $(p=0.003)$, sepsis $(p=0.045)$, right ventricular failure (RVF) $(p=0.007)$ and mesenteric ischemia $(p=0.044)$ than group $C$ and there was no difference between group $\mathrm{B}$ and $\mathrm{C}$ as regard incidence of complications. With respect to time to onset of complications, certain adverse events tended to occur in the first postoperative week (Table 6). These included renal failure, RVF and respiratory failure. Complications that were common one week to one month postoperatively were sepsis, respiratory failure followed by RVF. Cerebrovascular stroke was the most common complication after 6 months after the implantation.

\section{Postoperative outcome}

Length of stay in hospital and ICU was not significantly different between groups. However, duration of postoperative mechanical ventilation was longer in group A than group C (13.55 $\pm 13.43 \mathrm{vs}$ $6.80 \pm 12.75$ days, $p$ 0.006) and in group B than group C (12.61 \pm 24.93 vs $6.80 \pm 12.75$ days, $p$ 0.023). Left ventricular recovery was observed in 2 patients in group B. Thus, Weaning from VAD was possible. Myocarditis was present in both patients. The two patients represent $50 \%$ of BTR group. Mean survival of the entire cohort was $387.54 \pm 399.50$ days. Patients in group A survived shorter than group B (191.14 \pm 255.36 vs $505.87 \pm$ 482.91 days, $\mathrm{p}=0.003)$ and group $\mathrm{C}(191.14 \pm 255.36$ vs 378.93 \pm 282.48 days, $\mathrm{p}=0.009)$. Survival of the entire cohort was $64 \%$ at 3 months, $62 \%$ at 6 months and $60 \%$ at 1 year. At 3 months and 6 months, the survival rate was significantly higher in group $\mathrm{B}$ and $\mathrm{C}$ than group $\mathrm{A}$ and there was no difference in regard to 1-year survival between the groups. There was no difference in survival between group B and group C. 67 patients $(64.4 \%)$ of the entire cohort survived to discharge or to the end of the study. Survival to discharge was significantly lower in group A than B (11 patients, 40.7\%-33 patients, $70.2 \% \mathrm{p}=0.016$ ) and group C (11, 40.7\%-23, 76.7\% p=0.008) (Figure 2) (Table 7). Total mortality of the entire cohort was $47.1 \%$ (49 Patients). Total mortality of group A was significantly higher than group B (19 patients, $70.4 \%$ vs 19 patients, $40 \%, \mathrm{p}=0.017)$ and significantly higher than group $\mathrm{C}(19,70.4 \%$ vs $11,36.7 \%, \mathrm{p}=0.017)$. There was no significant difference in total mortality between group B and C. Early mortality (at 30 days after the operation) was 24 patients representing $23.1 \%$ of the entire cohort, it was significantly higher in group A than group B (12 patients, $44.4 \%$ vs 8 patients, $17 \%, \mathrm{p}=0.015)$ and group $\mathrm{C}(12,44.4 \%$ vs $4,13.3 \%$, $\mathrm{p}=0.010$ ), no difference was found in regard to early mortality between group B and C. In regard to late mortality, 25 patients 


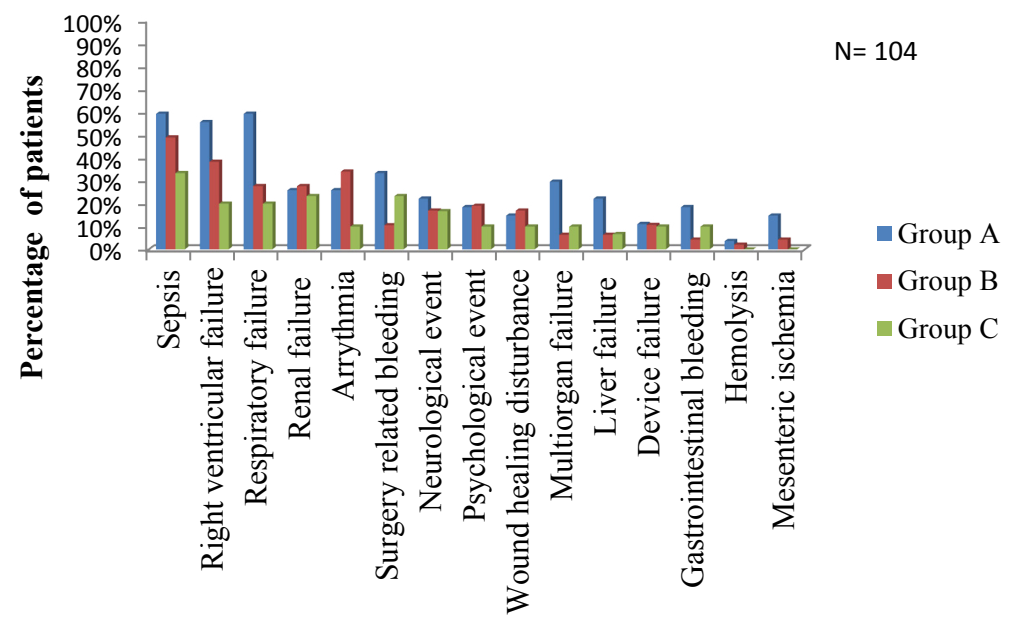

Postoperative complications

Figure 1. Postoperative complications split by INTERMCAS level (group A: level 1, group B: level 2/3, group C: level 4/5/6). Group A: INTERMACS 1, Group B: INTERMACS 2,3, Group C: INTERMACS 4-6, RV: Right Ventricular, GIT: Gastrointestinal Tract.

Table 6. Postoperative complications, split by their time of onset (number of patients=104).

\begin{tabular}{|c|c|c|c|c|c|c|c|}
\hline Type of complication & 1 week & 1week- 1 month & 1-3 months & 3-6 months & 6months-1 year & >1year & Total \\
\hline Sepsis $(\%)$ & 11.5 & 22.1 & 3.8 & 4.8 & 0 & 4.8 & 47.1 \\
\hline Right ventricular failure (\%) & 21.2 & 15.4 & 1 & 0 & 0 & 0 & 37.5 \\
\hline Respiratory failure (\%) & 16.3 & 16.3 & 1 & 0 & 0 & 0 & 33.7 \\
\hline Renal failure (\%) & 23.1 & 1.9 & 0 & 0 & 0 & 1 & 26 \\
\hline Tachyarrhythmia (\%) & 12.5 & 7.6 & 1.9 & 0 & 1 & 1.9 & 25 \\
\hline Surgery related bleeding (\%) & 13.5 & 4.8 & 1 & 1 & 0 & 0 & 20.2 \\
\hline Neurological event (\%) & 4.8 & 3.8 & 2.8 & 0 & 1.9 & 4.8 & 18.3 \\
\hline Psychological event (\%) & 11.5 & 4.8 & 0 & 0 & 0 & 0 & 16.3 \\
\hline Wound healing disturbance (\%) & 0 & 3.8 & 4.8 & 1.9 & 1.9 & 1.9 & 14.4 \\
\hline Multiorgan failure (\%) & 5.8 & 4.8 & 2.9 & 0 & 0 & 0 & 13.5 \\
\hline Device failure (\%) & 0 & 1.9 & 3.8 & 1.9 & 1 & 1.9 & 10.6 \\
\hline Liver failure (\%) & 6.7 & 2.9 & 1 & 0 & 0 & 0 & 10.6 \\
\hline $\begin{array}{l}\text { Gastrointestinal bleeding } \\
\text { bleeding (\%) }\end{array}$ & 1 & 3.8 & 4.8 & 0 & 0 & 0 & 9.6 \\
\hline Mesenteric ischemia (\%) & 2.9 & 2.9 & 0 & 0 & 0 & 0 & 5.8 \\
\hline Hemolysis (\%) & 1 & 0 & 0 & 0 & 0 & 1 & 1.9 \\
\hline
\end{tabular}

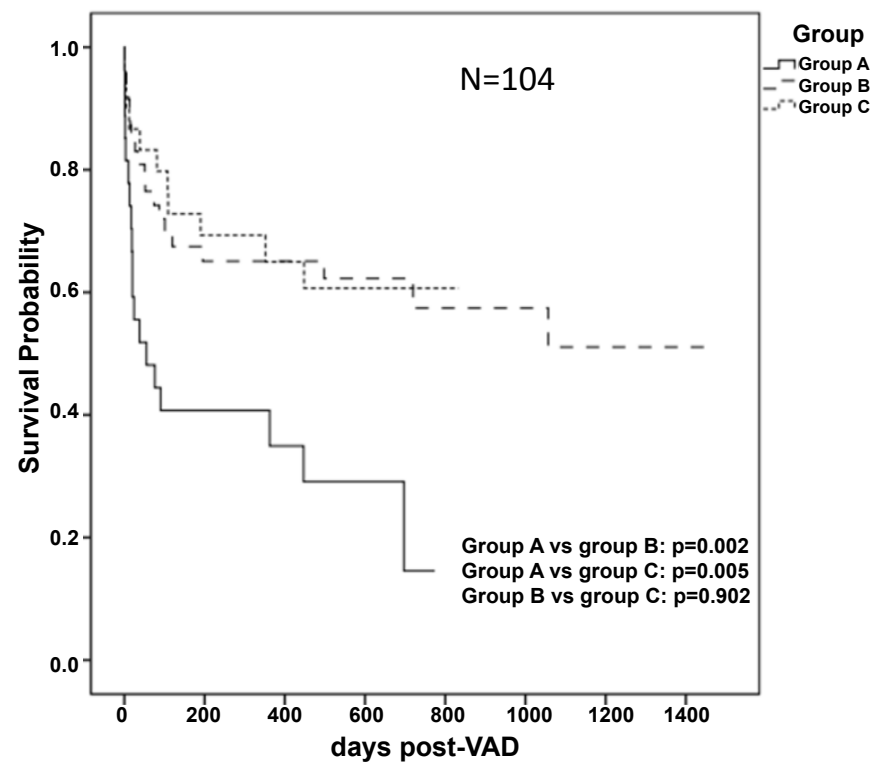

Figure 2. Kaplan Meier analysis of survival per INTERMCAS (group A: level 1, group B: level 2/3, group C: level 4/5/6). Number of patients=104. 
died after 30 days post implantation ( $24 \%$ of the entire cohort), and there was no significant difference between groups. Data for causes of death are given in (Table 8). Using cox regression analysis, we identified the preoperative predictors of total mortality (Table 9). Those variables reaching significance in invariable analysis were computed in multivariable regression. Continuous variables were dichotomized by multiple serial $\chi^{2}$ testing with stepwise threshold progression to identify optimal cut-offs for mortality prediction.

\section{Discussion}

We demonstrated that outcome and complications after LVAD implantation are strongly correlated to the preoperative status as described by the INTERMACS level. Preoperatively, group A demonstrated significant hemodynamic instability as well as a higher incidence of liver and renal impairment as well as lactic acidosis which indicates a hypo perfusion state in comparison with the other two groups. Moreover, there was a higher incidence of mechanical ventilation, ECMO therapy, CVVHD, IABP therapy and cardiopulmonary resuscitation in INTERMCAS 1 patients before implantation. The worse preoperative clinical and laboratory status of INTERMCAS 1 patients had a tremendous impact on the postoperative outcome. Our findings are in accordance with other studies $[6,8,9]$ Adverse events most frequently in our study population were sepsis $(47.1 \%)$, right ventricular failure $(37.5 \%)$, respiratory failure $(33.7 \%)$, renal failure $(26 \%)$, tachyarrhythmia $(25 \%)$ and surgery related bleeding $(20.2 \%)$. These were in accordance with several studies [8,10-12], though Strueber et al. [13] found the prevalence of neurological complications to be higher than that of right ventricular failure. Differences may be attributed to variability in defining events or to differences in patient population, which may also explain the differences in the events 'rate between the studies. Postoperative sepsis continues to be a problem in LVAD patients. The causes of sepsis can be divided into VAD specific, VAD related and non VAD related causes [14]. In our study, sepsis was attributed mostly to VAD non-related infections $(27.9 \%$ of the entire cohort) such as pneumonia, urinary tract infection, clostridium difficile associated diarrhea and peritonitis, whereas VAD specific infections including LVAD driveline infection occurred in $14.4 \%$ and VAD related infection such as blood borne infection affected only $4.8 \%$. Strueber et al. [13] also found pneumonia to be the most common cause of sepsis after HVAD implantation. In accordance with our results, Popov et al. [15] discovered $15 \%$ driveline infection rate in patients implanted with HVAD. Moreover, sepsis was most common after one week to one month after the procedure and affected 23 patients $22.1 \%$ in our investigation. The etiology of sepsis in this period was mostly due to VAD non related causes, which come in accordance with other studies [16-18] Sepsis was also the most common complication three to six months after surgery, however the main etiology of sepsis in this phase were VAD specific infections. This was in accordance with other studies $[16,18]$ and underlines the importance of careful mid- and long-term observation of infection signs particularly in the driveline site as well as sings of endocarditis. Mechanical complications occurred in 11 patients $10.6 \%$, and were divided to: pump thrombosis (6 patients, 5.8\%), driveline disconnection (4 patients, 3.8\%) and device failure (1 patient, 1\%). Pump thrombosis was treated medically by thrombolytic therapy using systemic Alteplase und was associated with $50 \%$ mortality, one event of thrombus recurrence was recorded. The rate of pump thrombosis in other studies that discussed CF-LVAD

Table 7. 3 months-, 6 months- and 1-year survival, also split by INTERMACS level (group A: level 1, group B: level 2/3, group C: level 4/5/6).

\begin{tabular}{|c|c|c|c|c|c|c|c|}
\hline Survival & Cohort (n=104) & Group A (n=27) & Group B (n=47) & Group C (n=30) & p value A vs B & p value A vs C & p value B vs C \\
\hline 3 months & $71(64 \%)$ & $12(44 \%)$ & $35(74 \%)$ & $24(80 \%)$ & 0.013 & 0.007 & 0.598 \\
\hline 6 months & $65(62 \%)$ & $11(41 \%)$ & $32(68 \%)$ & $22(73 \%)$ & 0.029 \\
\hline 1 year & $62(60 \%)$ & $11(41 \%)$ & $31(66 \%)$ & $20(67 \%)$ & 0.017 \\
\hline
\end{tabular}

Table 8. Causes of death, also split by INTERMACS level (group A: level 1, group B: level 2/3, group C: level 4/5/6.

\begin{tabular}{|c|c|c|c|}
\hline Cause of death & Cohort (n=104) & Group A (n=27) & Group B (n=47) \\
\hline Sepsis & $10(9.6 \%)$ & $3(11.1 \%)$ & $6(12.8 \%)$ \\
\hline Multiorgan failure & $14(13.5 \%)$ & $8(29.6 \%)$ & $3(6.4 \%)$ \\
\hline Right ventricular failure & $11(10.6 \%)$ & $4(14.8 \%)$ & $5(10.6 \%)$ \\
\hline Hemorrhagic stroke & $7(6.7 \%)$ & $4(14.8 \%)$ & $2(4.3 \%)$ \\
\hline Ischemic stroke & $1(1 \%)$ & $0(0 \%)$ & $0(0 \%)$ \\
\hline Device failure & $1(1 \%)$ & $0(0 \%)$ & $1(2.1 \%)$ \\
\hline Driveline disconnection & $1(1 \%)$ & $0(0 \%)$ & $1(2.1 \%)$ \\
\hline Pump thrombosis & $3(2.9 \%)$ & $0(0 \%)$ & $0(0 \%)$ \\
\hline Surgery related bleeding & $1(1 \%)$ & $0(0 \%)$ & $1(2.1 \%)$ \\
\hline Total & $49(47.1 \%)$ & $19(70.4 \%)$ & $19(40.4 \%)$ \\
\hline
\end{tabular}

Table 9. Preoperative predictors of total mortality in total cohort per Cox regression analysis.

\begin{tabular}{|c|c|}
\hline Predictor of overall mortality: & Cox regression Analysis: \\
\hline preoperative CVP $\geq 12 \mathrm{mmHg}$ & Hazard ratio $[\mathrm{HR}], 1.077 ; 95 \%$ confidence interval $[\mathrm{Cl}], 1.019-1.138 ; p=0.008$ \\
\hline preoperative systolic PAP $\geq 52 \mathrm{mmHg}$ & Hazard ratio [HR], 1.056; 95\% confidence interval [Cl], 1.015-1.099; $p=0.007$ \\
\hline high preoperative serum urea nitrogen $\geq 30 \mathrm{mg} / \mathrm{dl}$ & Hazard ratio $[\mathrm{HR}], 1.031 ; 95 \%$ confidence interval $[\mathrm{Cl}], 1.018-1.045 ; p=0.001$ \\
\hline high preoperative PCT $\geq 0.75 \mathrm{ng} / \mathrm{ml}$ & Hazard ratio $[\mathrm{HR}], 1.134 ; 95 \%$ confidence interval $[\mathrm{Cl}], 1.040-1.237 ; \mathrm{p}=0.04$ \\
\hline preoperative metabolic acidosis & Hazard ratio $[\mathrm{HR}], 3.496 ; 95 \%$ confidence interval $[\mathrm{Cl}], 1.708-7.157 ; p=0.001$ \\
\hline
\end{tabular}


implantation ranged from $2 \%$ to $26 \% \quad[13,16,19,20]$. Disconnection of the driveline which connects the pump to the controller and the battery causes immediate cessation of pump rotation occurred in 4 patients $(3.8 \%)$, necessitating cardiopulmonary resuscitation in 3 patients and directly causing mortality of one of these patients. The attempt to disconnect the driveline was identified as suicidal in 3 patients and accidental in one patient. There are few studies that discuss psychological complications after LVAD [21,22], one case report discussed a destination therapy patient who committed suicide by disconnecting his driveline [23]. VAD recipients are at high risk for depression and adjustment disorders, psychiatric conditions may be even more pronounced in destination therapy patients as they must comply with a complex medical regimen indefinitely. Device malfunction due to unexplained cessation of pump function was reported in one patient (1\%) after 3 years of implantation and was complicated by cardiogenic shock; the patient underwent LVAD exchange and died after 2 days due to multiorgan failure. Other Studies recorded device failure due manufacturing issues in HVAD system between $3 \%$ and $4 \%$ $[13,15]$ and it is in accordance with failure rates in other CFVAD devices [24]. Our data confirm INTERMACS data, which consistently demonstrates that critically ill patients (level 1) have the highest mortality [8]. The total mortality in group A was significantly higher than group B and C. In our study, this was attributed to the significantly higher early mortality in this group. However, there was no difference in regard to late mortality between the groups. In accordance with these results, Aaronson et al. [20] found that, most deaths after HVAD implantation occurred within the first 30 to 60 days post operatively [16,25]. Most of this early mortality has been attributed to patient selection, with the sickest patients having the worst outcome. This recognition highlights the importance of INTERMACS profiles as an important practical preoperative risk stratification tool. The main causes of mortality in our study were multiorgan failure, right ventricular failure and sepsis. In accordance with these results, Aaronson et al. [20] mentioned that, multiorgan failure is the most common cause of death after HVAD implantation. Others [12,26,27] also identified sepsis, right ventricular failure and multiorgan failure as the most common causes of death after CF-LVAD implantation. Alba, Rao et al. [11] identified multiorgan failure as the most common cause of death in INTERMCAS 1-2 patients and sepsis in INTERMCAS 3-4 patients. As these complications were unrelated to device malfunction, this finding suggests that selection of candidates and timing of LVAD implantation are the most likely determinants of the operative success. Interestingly, the most common cause of death in group $\mathrm{C}$ patients in our study was LVAD pump thrombosis which usually occurred in the late postoperative phase. This can be attributed to the better survival in this group which makes them more likely to experience this deadly complication. Survival results have shown a significant lower survival of patients with INTERMCAS 1 in comparison to the other INTERMCAS levels. The difference in survival was significant at 3 and 6 months but not at 1 year. Boyle et al [9] have shown lower actuarial survival and survival to discharge in patients at INTERMCAS 1 in comparison with patients at INTERMCAS
4, 5. The INTERMACS data emphasized that patients in the most critical condition at implant experienced worse survival than more stable patients, with early death accounting for the differences [7]. 6 months and 1-year survival ranged in other studies that addressed CF-LVAD between $70-91 \%$ and $62-86 \%$ respectively. $[7,8,10-13,16,19,24]$. These results are comparable to survival results of group $\mathrm{C}$ patients in our study. Our study does not demonstrate better results in terms of survival than other CF-LVAD clinical trials. At the time frame, covered by this analysis, it was the policy of our center to implant all patients suffering from end-stage refractory heart failure regardless of the severity. This should be taken into account, when comparing our data with other in clinical studies, which use more stringent inclusion and exclusion criteria. Predictors of mortality after LVAD Implantation were discussed in previous studies. We identified high preoperative serum urea nitrogen with cutoff $30 \mathrm{mg} / \mathrm{dl}$ as a predictor of total mortality. Preoperative renal impairment was identified as a predictor of mortality in other studies. Sandner et al. [28] found that, the survival rates of patients whose Glomerular Filtration Rate (GFR) was $<60 \mathrm{~mL} / \mathrm{min} / 1.73 \mathrm{~m}^{2}$ at LVAD implantation were significantly worse than those with GFR $>60 \mathrm{~mL} / \mathrm{min} / 1.73 \mathrm{~m}^{2}$. In addition, Butler et al. [29] reported on patients with creatinine clearance $(\mathrm{CrCl})$ values of $<47 \mathrm{ml} / \mathrm{min}$ to have a significantly higher risk of mortality than patients with $\mathrm{CrCl}$ values of $>95$ $\mathrm{ml} / \mathrm{min}$ (OR:1.95;95\% CI, 1.14-3.63). We also have identified a high preoperative central venous pressure with cutoff $12 \mathrm{mmHg}$ and a high systolic pulmonary artery pressure with cutoff 52 $\mathrm{mmHg}$ to be predictors of total mortality. The data from several sources have identified the role of central venous pressure (CVP) as a critical hemodynamic parameter in LVAD-patients. Sabashnikov et al. [30] found that, the only influenceable independent risk factor predictive of the 90-day mortality in their cohort was the preoperative CVP, with cutoff $18 \mathrm{mmHg}$. Rao, Oz et al. [4] identified preoperative CVP with a cutoff 16 $\mathrm{mmHg}$. Preoperative sepsis as a risk factor of decreased survival after LVAD implantation has been discussed in few studies [31]. We identified a high preoperative procalcitonin, which is a well-known specific and sensitive sepsis biomarker, with a cutoff value $0.75 \mathrm{ng} / \mathrm{ml}$ as risk factor for mortality. [32] In their preoperative risk prediction model, Klotz et al. [6] identified white blood cell count $>13 \times 10^{3} \mu / \mathrm{L}$ and C-reactive protein $>8$ $\mathrm{mg} / \mathrm{dl}$ among other factors as significant mortality risk factors.

\section{Study Limitations and Strengths}

The main limitation of our study is its retrospective design, lack of randomization and limitation to a single institution. On the other hand, the paper is strong in terms of complete data collection and structured analysis of complications in the postoperative course combined with reliable statistical analysis.

\section{Conclusion}

LVAD implantation under stable conditions warrants a good prognosis for patients with severe heart failure. The outcome of INTERMACS level 1 patients is worse than other levels. The most influential preoperative measures to improve survival after LVAD implantation are lowering pulmonary vascular resistance to optimize right-heart function, reduce right atrial 
Citation: Ahmed AR, Pizanis N, Kamler M, et al. Survival analysis and postoperative complications after ventricular assist device implantation, prognostic value of INTERMACS scale. Ann Cardiovasc Thorac Surg. 2018;1(1):15-23.

pressure and secondary hepatic congestion, aggressive volume management to minimize right ventricular workload, optimize renal function, optimize acid base balance and preoperative sepsis management [33]. We consider LVAD Implantation still important to save a significant percentage of these otherwise dying patients in INTERMACS 1 group. To further improve the results for these critically ill patients, measures such as bridging with intermediate term treatment uni-or biventricular extracorporeal life support systems and implantation of the VAD after hemodynamic stabilization should be implemented.

\section{References}

1. Rose EA, Gelijns AC, Moskowitz AJ, et al. Long-term use of a left ventricular assist device for end-stage heart failure. N Engl J Med. 2001;345:1435-43.

2. Stehlik J, Rahmel AO, Hertz MI, et al. The Registry of the International Society for heart and lung transplantation: twenty-seventh official adult heart transplant report--2010. J Heart Lung Transplant. 2010;29:1089-103.

3. Jaski BE, Kim JC, Naftel DC, et al. Cardiac transplant outcome of patients supported on left ventricular assist device vs. intravenous inotropic therapy. J Heart Lung Transplant. 2001;20:449-56.

4. Rao V, Oz MC, Flannery MA, et al. Revised screening scale to predict survival after insertion of a left ventricular assist device. J Thorac Cardiovasc Surg. 2003;125:855-62.

5. Deng MC, Loebe M, El-Banayosy A, et al. Mechanical circulatory support for advanced heart failure: effect of patient selection on outcome. Circulation. 2001;103:231-7.

6. Klotz S, Vahlhaus C, Riehl C, et al. Pre-operative prediction of post-VAD implant mortality using easily accessible clinical parameters. J Heart Lung Transplant. 2010;29:4552 .

7. Kirklin JK, Naftel DC, Stevenson LW, et al. INTERMACS database for durable devices for circulatory support: first annual report. J Heart Lung Transplant. 2008;27:1065-72.

8. Kirklin JK, Naftel DC, Pagani FD, et al. Sixth INTERMACS annual report: a 10,000-patient database. J Heart Lung Transplant. 2014;33:555-64.

9. Boyle AJ, Ascheim DD, Russo MJ, et al. Clinical outcomes for continuous-flow left ventricular assist device patients stratified by pre-operative INTERMACS classification. J Heart Lung Transplant. 2011;30:402-7.

10. Yuan N, Arnaoutakis GJ, George TJ, et al. The spectrum of complications following left ventricular assist device placement. J Card Surg. 2012;27:630-8.

11. Alba AC, Rao V, Ivanov J, et al. Usefulness of the INTERMACS scale to predict outcomes after mechanical assist device implantation. J Heart Lung Transplant. 2009;28:827-33.

12. Wu L, Weng YG, Dong NG, et al. Outcomes of heartware ventricular assist system support in 141 patients: a singlecentre experience. Eur J Cardiothorac Surg. 2013;44:13945.
13. Strueber M, O'Driscoll G, Jansz P, et al. Multicenter evaluation of an intrapericardial left ventricular assist system. J Am College Cardiol. 2011;57:1375-82.

14. Hannan MM, Husain S, Mattner F, et al. Working formulation for the standardization of definitions of infections in patients using ventricular assist devices. J Heart Lung Transplant. 2011;30:375-84.

15. Popov AF, Hosseini MT, Zych B, et al. Clinical experience with HeartWare left ventricular assist device in patients with end-stage heart failure. Ann Thorac Surg, 2012;93:810-5.

16. Miller LW, Pagani FD, Russell SD, et al. Use of a continuousflow device in patients awaiting heart transplantation. $\mathrm{N}$ Engl J Med. 2007;357:885-96.

17. Holman WL, Park SJ, Long JW, et al. Infection in permanent circulatory support: Experience from the REMATCH trial. J Heart Lung Transplant. 2004;23:1359-65.

18. Topkara VK, Kondareddy S, Malik F, et al. Infectious complications in patients with left ventricular assist device: etiology and outcomes in the continuous-flow era. Ann Thorac Surg. 2010;90:1270-7.

19. Wieselthaler GM, Gerry O, Jansz P, et al. Initial clinical experience with a novel left ventricular assist device with a magnetically levitated rotor in a multi-institutional trial. J Heart Lung Transplant. 2010;29:1218-25.

20. Aaronson KD, Slaughter MS, Miller LW, et al. Use of an intrapericardial, continuous-flow, centrifugal pump in patients awaiting heart transplantation. Circulation. 2012;125:3191-200.

21. Baba A, Hirata G, Yokoyama F, et al. Psychiatric problems of heart transplant candidates with left ventricular assist devices. J Artif Organs. 2006;9:203-8.

22. Casida JM, Parker J. A preliminary investigation of symptom pattern and prevalence before and up to 6 months after implantation of a left ventricular assist device. J Artif Organs. 2012;15:211-4.

23. Tigges-Limmer K, Schönbrodt M, Roefe D, et al. Suicide after ventricular assist device implantation. J Heart Lung Transplant. 2010;29:692-4.

24. Pagani FD, Miller LW, Russell SD, et al. Extended mechanical circulatory support with a continuous-flow rotary left ventricular assist device. J Am College Cardiol. 2009;54:312-21.

25. Slaughter MS, Rogers JG, Milano CA, et al. Advanced heart failure treated with continuous-flow left ventricular assist device. N Engl J Med. 2009;361:2241-51.

26. Lietz K, Long JW, Kfoury AG, et al. Outcomes of left ventricular assist device implantation as destination therapy in the post-REMATCH era: implications for patient selection. Circulation. 2007;116:497-505.

27. Kirklin JK, Naftel DC, Pagani FD, et al. Long-term mechanical circulatory support (destination therapy): on track to compete with heart transplantation?. J Thorac Cardiovasc Surg. 2012;144:584-603. 
28. Sandner SE, Zimpfer D, Zrunek P, et al. Renal function and outcome after continuous flow left ventricular assist device implantation. Ann Thorac Surg. 2009;87:1072-8.

29. Butler J, Geisberg C, Howser R, et al., Relationship between renal function and left ventricular assist device use. Ann Thorac Surg. 2006;81:1745-51.

30. Sabashnikov A, Mohite PN, Zych B, et al. Outcomes and predictors of early mortality after continuous-flow left ventricular assist device implantation as a bridge to transplantation. Asaio J. 2014;60:162-9.
31. Stevenson LW, Pagani FD, Young JB, et al. INTERMACS profiles of advanced heart failure: The current picture. $\mathrm{J}$ Heart Lung Transplant. 2009;28:535-41.

32. Balk RA, Cerra FB, Dellinger RP, et al. Definitions for sepsis and organ failure and guidelines for the use of innovative therapies in sepsis. Crit Care Med. 1992;20:864-74.

33. Genovese EA, Dew MA, Teuteberg JJ, et al. Incidence and patterns of adverse event onset during the first 60 days after ventricular assist device implantation. Ann Thorac Surg. 2009;88:1162-70.

\section{*Correspondence to:}

Kamler Markus

Department of Thoracic and Cardiovascular Surgery University Hospital Essen

Hufelandstrasse

Essen

Germany

Tel: +49 2017232266

Email: markus.kamler@uk-essen.de 